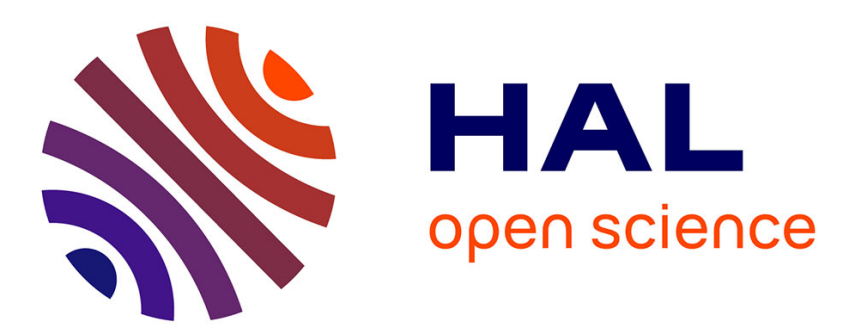

\title{
Nonlinear control of a coupled PDE/ODE system modeling a switched power converter with a transmission line
}

\author{
Jamal Daafouz, Marius Tucsnak, Julie Valein
}

\section{- To cite this version:}

Jamal Daafouz, Marius Tucsnak, Julie Valein. Nonlinear control of a coupled PDE/ODE system modeling a switched power converter with a transmission line. Systems and Control Letters, 2014, 70, pp.92-99. 10.1016/j.sysconle.2014.05.009 . hal-01095237

\section{HAL Id: hal-01095237 \\ https://hal.science/hal-01095237}

Submitted on 15 Dec 2014

HAL is a multi-disciplinary open access archive for the deposit and dissemination of scientific research documents, whether they are published or not. The documents may come from teaching and research institutions in France or abroad, or from public or private research centers.
L'archive ouverte pluridisciplinaire HAL, est destinée au dépôt et à la diffusion de documents scientifiques de niveau recherche, publiés ou non, émanant des établissements d'enseignement et de recherche français ou étrangers, des laboratoires publics ou privés. 


\title{
Nonlinear control of a coupled PDE/ODE system modelling a switched power converter with a transmission line
}

\author{
Jamal Daafouz, Marius Tucsnak and Julie Valein*
}

September 18, 2013

\begin{abstract}
We consider an infinite dimensional system modelling a boost converter connected to a load via a transmission line. The governing equations form a system coupling the telegraph partial differential equation with the ordinary differential equations modeling the converter. The coupling is given by the boundary conditions and the nonlinear controller we introduce. We design a nonlinear saturating control law using a Lyapunov function for the averaged model of the system. The main results give the well-posedness and stability properties of the obtained closed loop system.
\end{abstract}

Keywords: infinite dimensional system, telegraph equations, power converters, stabilization, coupled system.

\section{Introduction and main results}

Systems coupling partial and ordinary differential equations have been intensively studied from a control theoretical view point, namely due to their applications to the stabilization of flexible structures (we refer to Littman and Markus [10] for an early contribution on the subject and to Weiss and Zhao [22] for a description of recent works in the area). More recently, such systems appeared in the context of energy management and more precisely in modeling power converters connected to transmission lines (see, for instance, Zainea, Van der Schaft and Buisson [21] and references therein). From a mathematical view point, converters are primarily modeled by ordinary differential equations where the input takes values in a discrete set (for instance $\{0,1\})$. This fact leads to hybrid models, in the sense that they couple continuous and discrete dynamics [8]. To avoid this difficulty, a classical approach used in applications for many years assumes that the control law is computed using an averaging method, so that the considered control is now with continuous values [14,17]. Several approaches have been proposed in the literature such as passivity based methods [19] and sliding modes techniques [18]. Recently, hybrid approaches have been proposed for stabilization and optimal control of these devices $[7,5,12]$ or for the determination of optimal switching instants posed as a non-smooth dynamic optimization problem [11]. We adopt below the classical "averaging" approach, so that the controlled converter

*J. Daafouz is with CRAN UMR 7039, Université de Lorraine/CNRS, 2 av. de la forêt de Haye, 54516 Vandœuvre-lès-Nancy Cedex, France. M. Tucsnak and J. Valein are with Institut Élie Cartan, UMR 7502, Université de Lorraine/CNRS, POB239, 54506 Vandœuvre-lèsNancy Cedex, France. jamal daafouz@univ-lorraine.fr, marius.tucsnak@univ-lorraine.fr, julie.valein@univ-lorraine.fr 
is modeled by a system of ODE's with a control acting on one of the coefficients. This nonlinear and saturating control law is built using a Lyapunov function. The obtained equations have common points with infinite dimensional bilinear control systems such as those considered in the classical works of Ball and Slemrod [3], Ball, Marsden and Slemrod [2] or more recently in the context of quantum control (see, for instance, Beauchard and Mirrahimi [4] or Amini, Mirrahimi and Rouchon [1]). In this work we take advantage of the fact that the feedback control appears in the finite dimensional part of the system so that we are able to provide a strong stability result, even in the case of a saturating feedback law.

The system of coupled partial and ordinary differential equations which will be studied in the work describes the coupling of a boost converter and a transmission line which is described in Figure 1. Moreover, to take in consideration that the physical control cannot lie outside $[0,1]$, we used a saturating feedback law.

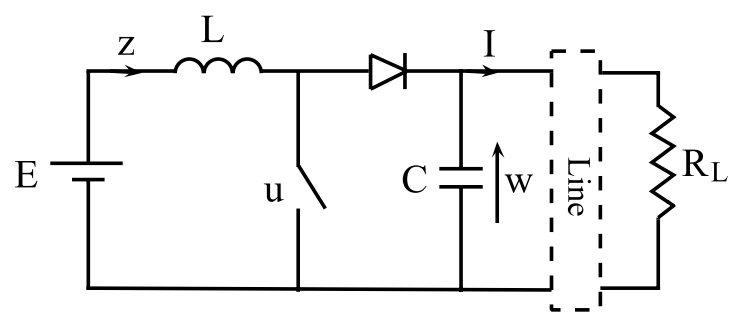

Figure 1: Power converter with a transmission line

Denoting by $z(t)$ the inductor current and by $w(t)$ the capacitor voltage, it is well known that the converter is described by the ordinary differential equations:

$$
\begin{gathered}
\dot{z}(t)=-\frac{\rho(t)}{L} w(t)+\frac{E}{L}, \\
\dot{w}(t)=\frac{\rho(t)}{C} z(t)-\frac{1}{C} I(0, t),
\end{gathered}
$$

where $I(0, t)$ is the current at the "left end" of the transmission line, the function $\rho:[0, \infty) \rightarrow[0,1]$ is the input, $E>0$ is a constant source voltage, $L>0$ is the inductance and $C>0$ is the capacitance. $\rho=0$ corresponds to the switch being closed and $\rho=1$ corresponds to it being open. The averaged character of our model (corresponding to a very high switching frequency) is expressed by the fact that the control $\rho$ takes values in the interval $[0,1]$ instead of the discrete set $\{0,1\}$ which is generally associated to the switch configuration. Such an "average model" is classical in the literature and it means that the control to be designed is not the switching signal corresponding to the position of the switch in the circuit but the so called duty cycle, which is the average of this switching signal over a short time interval. Typically, this time interval is the switching period. The current $I$ and the voltage $V$ in the transmission line satisfy the telegraph equations

$$
\begin{gathered}
\frac{\partial I}{\partial t}(x, t)=-L_{l}^{-1} \frac{\partial V}{\partial x}(x, t) \quad(x \in(0,1), t \geqslant 0), \\
\frac{\partial V}{\partial t}(x, t)=-C_{l}^{-1} \frac{\partial I}{\partial x}(x, t) \quad(x \in(0,1), t \geqslant 0), \\
V(0, t)=w(t), V(1, t)=R_{L} I(1, t) \quad(t \geqslant 0) .
\end{gathered}
$$

The system which is studied in this paper is formed by equations (1.1)-(1.5), together with the initial conditions

$$
z(0)=z_{0}, \quad w(0)=w_{0},
$$




$$
I(x, 0)=I_{0}(x), \quad V(x, 0)=V_{0}(x) \quad(x \in(0,1)) .
$$

Here $L_{l}$ and $C_{l}$ are positive constants that characterize the transmission line and $R_{L}$ is the load resistance at the end of the line.

Our main result concerns the design of a feedback control for (1.1)-(1.7), which steers the system to an admissible reference state. Recall that admissible reference states are time independent solutions of (1.1)-(1.7). In our case, an admissible reference state is a quadruple

$$
\left[\begin{array}{c}
z^{*} \\
w^{*} \\
I^{*} \\
V^{*}
\end{array}\right] \in \mathbb{R} \times \mathbb{R} \times L^{2}[0,1] \times L^{2}[0,1]
$$

such that

$$
\begin{gathered}
-\frac{\rho^{*}}{L} w^{*}+\frac{E}{L}=0, \\
\frac{\rho^{*}}{C} z^{*}-\frac{1}{C} I^{*}(0)=0, \\
-L_{l}^{-1} \frac{\partial V^{*}}{\partial x}(x)=0 \quad(x \in(0,1)), \\
-C_{l}^{-1} \frac{\partial I^{*}}{\partial x}(x)=0 \quad(x \in(0,1)), \\
V^{*}(0)=w^{*}, V^{*}(1)=R_{L} I^{*}(1),
\end{gathered}
$$

for some $\rho^{*} \in(0,1)$. It is easy to check that, given $\rho^{*} \in(0,1)$ the unique solution of (1.8)-(1.12) (thus our admissible reference state) is given by

$$
\begin{gathered}
z^{*}=\frac{E}{\rho^{* 2} R_{L}}, \quad w^{*}=\frac{E}{\rho^{*}}, \\
I^{*}(x)=\frac{E}{\rho^{*} R_{L}}, \quad V^{*}(x)=\frac{E}{\rho^{*}} \quad(x \in(0,1)) .
\end{gathered}
$$

The control problem we consider consists, given $\rho^{*} \in(0,1)$, in finding a feedback law for the control $\rho$ in order to have (in an appropriate sense)

$$
\lim _{t \rightarrow \infty}\left[\begin{array}{c}
z(t) \\
w(t) \\
I(t) \\
V(t)
\end{array}\right]=\left[\begin{array}{c}
z^{*} \\
w^{*} \\
I^{*} \\
V^{*}
\end{array}\right] .
$$

In order to design this feedback law we use a simple calculation, assuming that (1.1)(1.7) admits smooth solutions. More precisely, we assume that $z, w, I, V$ are smooth enough and that they satisfy (1.1)-(1.7). Consider the "energy" function $W: \mathbb{R} \times \mathbb{R} \times$ $L^{2}[0,1] \times L^{2}[0,1] \rightarrow \mathbb{R}$, defined by

$$
\begin{array}{r}
W\left(\begin{array}{c}
z \\
w \\
I(\cdot) \\
V(\cdot)
\end{array}\right)=L\left|z-z^{*}\right|^{2}+C\left|w-w^{*}\right|^{2}+L_{l}\left\|I(\cdot)-I^{*}\right\|_{L^{2}[0,1]}^{2}+C_{l}\left\|V(\cdot)-V^{*}\right\|_{L^{2}[0,1]}^{2} \\
\left(z, w \in \mathbb{R}, \quad I, \quad V \in L^{2}[0,1]\right) .
\end{array}
$$


A simple calculation shows that

$$
\begin{array}{r}
\frac{1}{2} \frac{\mathrm{d}}{\mathrm{dt}} W\left(\begin{array}{c}
z \\
w \\
I(\cdot) \\
V(\cdot)
\end{array}\right)=L \dot{z}(t)\left(z(t)-z^{*}\right)+C \dot{w}(t)\left(w(t)-w^{*}\right)+L_{l} \int_{0}^{1} \frac{\partial I}{\partial t}(x, t)\left(I(x, t)-I^{*}\right) \mathrm{d} x \\
+C_{l} \int_{0}^{1} \frac{\partial V}{\partial t}(x, t)\left(V(x, t)-V^{*}\right) \mathrm{d} x
\end{array}
$$

Using (1.1)-(1.4), we have

$$
\begin{gathered}
\frac{1}{2} \frac{\mathrm{d}}{\mathrm{dt}} W\left(\begin{array}{c}
z \\
w \\
I(\cdot) \\
V(\cdot)
\end{array}\right)=\rho(t)\left(w(t) z^{*}-w^{*} z(t)\right)+E\left(z(t)-z^{*}\right)-I(0, t) w+I(0, t) w^{*} \\
-\int_{0}^{1}\left(\frac{\partial V}{\partial x}(x, t) I(x, t)+\frac{\partial I}{\partial x}(x, t) V\right) \mathrm{d} x+I^{*} \int_{0}^{1} \frac{\partial V}{\partial x}(x, t) \mathrm{d} x+V^{*} \int_{0}^{1} \frac{\partial I}{\partial x}(x, t) \mathrm{d} x .
\end{gathered}
$$

With integrations by parts and (1.5), we obtain

$$
\begin{array}{r}
\frac{1}{2} \frac{\mathrm{d}}{\mathrm{dt}} W\left(\begin{array}{c}
z \\
w \\
I(\cdot) \\
V(\cdot)
\end{array}\right)=\rho(t)\left(w(t) z^{*}-z(t) w^{*}\right)+E\left(z(t)-z^{*}\right)+I(0, t)\left(w^{*}-V^{*}\right) \\
-R_{L}|I(1, t)|^{2}+I(1, t)\left(R_{L} I^{*}+V^{*}\right)-I^{*} w(t) .
\end{array}
$$

Then (1.13)-(1.14) give, for every $t \geqslant 0$,

$$
\frac{\mathrm{d}}{\mathrm{dt}} W\left(\begin{array}{c}
z \\
w \\
I(\cdot) \\
V(\cdot)
\end{array}\right)=-2 R_{L}\left|I(1, t)-I^{*}\right|^{2}+2\left(\rho^{*}-\rho(t)\right)\left(w^{*} z(t)-w(t) z^{*}\right) .
$$

We emphasize that, at this stage, formula (1.16) is not rigourously proved. We also remark that if there are no disturbances acting on our system then we could choose a constant $\rho=\rho^{*}$ that leads to the desired equilibrium output voltage $w=\frac{E}{\rho^{*}}$, according to (1.13). Indeed, for a constant $\rho$ the system would become linear and stable. However, in applications, there are always disturbances added to the input voltage $E$ and the load current $I$, and maintaining a desired $w$ in spite of these disturbances is a difficult problem discussed in many references (see, for instance, [16]). The difficulty stems from the fact that the zero dynamics of the system is unstable. This is why we propose below a nonlinear control which offers the guarantees of the closed loop solution, namely robustness with respect to disturbances and parameter uncertainties. Moreover, formula (1.16) suggests that the nonlinear control below accelerates the decay of $W$.

According to (1.16), since we need $\rho(t) \in[0,1]$, a natural candidate for the feedback law is given by

$$
\rho(t):= \begin{cases}\rho^{*}+w^{*} z(t)-z^{*} w(t) & \text { if } \rho^{*}+w^{*} z(t)-z^{*} w(t) \in[0,1] \\ 0 & \text { if } \rho^{*}+w^{*} z(t)-z^{*} w(t)<0 \\ 1 & \text { if } \rho^{*}+w^{*} z(t)-z^{*} w(t)>1\end{cases}
$$

With the above choice for $\rho$, formula (1.16) implies that $W$ is a Lyapunov function for (1.1)-(1.7). 
The main results in this work concern the closed loop problem formed by (1.1)-(1.7) and (1.17). We obtain in this way a stability problem for a system coupling ODE's and PDE's with the dissipation acting via one of the coefficients in the ODE part. In this case of purely PDE systems this kind of stability question has been investigated, in particular, in $[3,13,20]$.

Our first main result asserts that the closed loop problem obtained by taking $\rho$ as in (1.17) is well-posed and satisfies an energy balance.

Theorem 1.1. For every $z_{0}, w_{0} \in \mathbb{R}$ and $I_{0}, V_{0} \in L^{2}[0,1]$ there exists a unique solution of (1.1)-(1.7), with $\rho$ given by (1.17). Moreover, if $z^{*}, w^{*}, I^{*}$ and $V^{*}$ are given by (1.13) and (1.14) and $W$ is defined by (1.15), this solution satisfies, for $t \geqslant 0$,

$$
\begin{aligned}
W\left(\begin{array}{c}
z \\
w \\
I(\cdot) \\
V(\cdot)
\end{array}\right)+2 R_{L} \int_{0}^{t}\left|I(1, \sigma)-I^{*}\right|^{2} \mathrm{~d} \sigma \\
\quad-2 \int_{0}^{t}\left(\rho^{*}-\rho(\sigma)\right)\left(w^{*} z(\sigma)-w(\sigma) z^{*}\right) \mathrm{d} \sigma=W\left(\begin{array}{c}
z_{0} \\
w_{0} \\
I_{0}(\cdot) \\
V_{0}(\cdot)
\end{array}\right) .
\end{aligned}
$$

Remark 1.2. Theorem 1.1 implies that $I(1, \cdot)$ belongs to $L^{2}([0, \infty))$. This can be seen as a "hidden" regularity property, in the sense of Lions [9].

Finally we show that under the action of the feedback law (1.17) the state of the system converges to the admissible reference state.

Theorem 1.3. Under the assumptions of Theorem 1.1, the solution of (1.1)-(1.7), with $\rho$ given by (1.17) and with $z_{0}, w_{0} \in \mathbb{R}, I_{0}, V_{0} \in H^{1}(0,1)$ with $V_{0}(0)=w_{0}$, $V_{0}(1)=R_{L} I_{0}(1)$, satisfies

$$
\lim _{t \rightarrow \infty} z(t)=z^{*}, \quad \lim _{t \rightarrow \infty} w(t)=w^{*} .
$$

and

$$
\lim _{t \rightarrow \infty} I(t)=I^{*}, \quad \lim _{t \rightarrow \infty} V(t)=V^{*},
$$

in $L^{2}[0,1]$.

The outline of this paper is as follows. In Section 2, we prove the main wellposedness result. The stability result is proved in Section 3.

\section{Well-posedness}

We first introduce some notation. Set

$$
Z=\left[\begin{array}{c}
z \\
w \\
I \\
V
\end{array}\right], \quad Z_{0}=\left[\begin{array}{c}
z_{0} \\
w_{0} \\
I_{0} \\
V_{0}
\end{array}\right]
$$

and consider the Hilbert space $X$ defined by

$$
X=\mathbb{R} \times \mathbb{R} \times L^{2}[0,1] \times L^{2}[0,1] .
$$

We endow $X$ with the norm (equivalent to the usual one)

$$
\|Z\|^{2}=L|z|^{2}+C|w|^{2}+L_{l}\|I\|_{L^{2}[0,1]}^{2}+C_{l}\|V\|_{L^{2}[0,1]}^{2} .
$$


We define the operators $A: \mathcal{D}(A) \rightarrow X$ and $P: X \rightarrow X$ by

$$
A Z=\left[\begin{array}{c}
0 \\
-\frac{I(0)}{C} \\
-\frac{1}{L_{l}} \frac{\mathrm{d} V}{\mathrm{~d} x} \\
-\frac{1}{C_{l}} \frac{\mathrm{d} I}{\mathrm{~d} x}
\end{array}\right], \quad P Z=\left[\begin{array}{c}
-\frac{w}{L} \\
\frac{z}{C} \\
0 \\
0
\end{array}\right],
$$

with

$$
\mathcal{D}(A)=\left\{Z \in X \mid I, V \in H^{1}(0,1), V(0)=w, V(1)=R_{L} I(1)\right\},
$$

where $H^{1}(0,1)$ stands, as usual practice, for the space of absolutely continuous functions on the interval $(0,1)$ whose derivative is in $L^{2}[0,1]$.

We have the following lemma.

Lemma 2.1. The operator $A$ is $m$-dissipative.

Proof. We first note that for every $Z \in \mathcal{D}(A)$ we have

$$
\langle A Z, Z\rangle=-I(0) w-\int_{0}^{1}\left(\frac{\mathrm{d} V}{\mathrm{~d} x} I+\frac{\mathrm{d} I}{\mathrm{~d} x} V\right) \mathrm{d} x .
$$

Using next the boundary condition (1.5) it easily follows

$$
\langle A Z, Z\rangle=-R_{L}|I(1)|^{2} \leqslant 0,
$$

so that $A$ is dissipative.

According to classical results, to show the m-dissipativity of $A$ it suffices to check that $\lambda I-A$ is onto for some $\lambda>0$. Therefore, given $\left(f_{1}, f_{2}, g_{1}, g_{2}\right)^{t} \in X$, we want to prove the existence of $Z=(z, w, I, V)^{t} \in \mathcal{D}(A)$ such that

$$
(\lambda I-A)\left[\begin{array}{c}
z \\
w \\
I \\
V
\end{array}\right]=\left[\begin{array}{l}
f_{1} \\
f_{2} \\
g_{1} \\
g_{2}
\end{array}\right] .
$$

The above system writes

$$
\left\{\begin{array}{l}
\lambda z=f_{1} \\
\lambda w+\frac{I(0)}{C}=f_{2} \\
\lambda I+\frac{1}{L_{l}} \frac{\mathrm{d} V}{\mathrm{~d} x}=g_{1} \\
\lambda V+\frac{1}{C_{l}} \frac{\mathrm{d} I}{\mathrm{~d} x}=g_{2},
\end{array}\right.
$$

which leads to

$$
\begin{gathered}
z=\frac{f_{1}}{\lambda}, \\
w=\frac{1}{\lambda}\left(f_{2}-\frac{I(0)}{C}\right) .
\end{gathered}
$$

We have to solve the differential equations

$$
\begin{aligned}
& \lambda I+\frac{1}{L_{l}} \frac{\mathrm{d} V}{\mathrm{~d} x}=g_{1}, \\
& \lambda V+\frac{1}{C_{l}} \frac{\mathrm{d} I}{\mathrm{~d} x}=g_{2},
\end{aligned}
$$

with the conditions $V(0)=w$ and $V(1)=R_{L} I(1)$. Differentiating the first equation, substituting $\frac{\mathrm{d} I}{\mathrm{~d} x}$ from the second one and using the fact that

$$
I=\frac{1}{\lambda}\left(g_{1}-\frac{1}{L_{l}} \frac{\mathrm{d} V}{\mathrm{~d} x}\right)
$$


lead to

$$
\left\{\begin{array}{l}
\lambda^{2} V-\frac{1}{C_{l} L_{l}} \frac{\mathrm{d}^{2} V}{\mathrm{~d} x^{2}}=\lambda g_{2}-\frac{1}{C_{l}} \frac{\mathrm{d} g_{1}}{\mathrm{~d} x} \in H^{-1}(0,1) \\
V(0)-\frac{1}{L_{l} \lambda^{2} C} \frac{\mathrm{d} V}{\mathrm{~d} x}(0)=\frac{1}{\lambda}\left(f_{2}-\frac{g_{1}(0)}{C \lambda}\right) \\
V(1)+\frac{R_{L}}{\lambda L_{l}} \frac{\mathrm{d} V}{\mathrm{~d} x}(1)=\frac{R_{L}}{\lambda} g_{1}(1)
\end{array} .\right.
$$

It is not difficult to check that the above system admits a unique solution $V \in H^{1}(0,1)$. Then we define $I$ by (2.6) and $w, z$ by (2.5) and (2.4). Setting $Z=(z, w, I, V)^{t}$, we see that $Z \in \mathcal{D}(A)$ is solution of (2.3), which finishes the proof of this lemma.

Let $F \in X$ be defined by

$$
F=\left[\begin{array}{c}
\frac{E}{L} \\
0 \\
0 \\
0
\end{array}\right]
$$

where $E$ and $L$ are the constants introduced in (1.1).

With the above notation, equations (1.1)-(1.7) can be rewritten as a bilinear control system of the form

$$
\dot{Z}(t)=A Z(t)+\rho(t) P Z(t)+F
$$

To study the existence and uniqueness of the closed loop problem, with $\rho$ given by (1.17), we first give the following local in time existence result:

Proposition 2.2. Let $R>0$. Then there exists $T_{\max }>0$, depending only on $R$, such that, for every $Z_{0} \in \mathcal{D}(A)$ such that $\left\|Z_{0}\right\| \leqslant R$ and every $T \in\left[0, T_{\max }\right)$, there exists a unique strong solution

$$
Z \in C([0, T], \mathcal{D}(A)) \cap C^{1}([0, T], X)
$$

of (2.7) with $\rho(t)$ given by (1.17).

On the other hand, for every $Z_{0} \in X$ such that $\left\|Z_{0}\right\| \leqslant R$ and every $T \in\left[0, T_{\max }\right)$, there exists a unique mild solution $Z \in C([0, T], X)$ of $(2.7)$ with $\rho(t)$ given by $(1.17)$. Moreover, if $z^{*}, w^{*}, I^{*}$ and $V^{*}$ are given by (1.13), (1.14) and $W$ is defined by (1.15), this solution satisfies

$$
\begin{aligned}
W(Z(t))+ & 2 R_{L} \int_{0}^{t}\left|I(1, \sigma)-I^{*}\right|^{2} \mathrm{~d} \sigma \\
& -2 \int_{0}^{t}\left(\rho^{*}-\rho(\sigma)\right)\left(w^{*} z(\sigma)-w(\sigma) z^{*}\right) \mathrm{d} \sigma=W\left(Z_{0}\right) \quad(t \in[0, T]) .
\end{aligned}
$$

Proof. For every

$$
Z=\left[\begin{array}{c}
z \\
w \\
I \\
V
\end{array}\right] \in X
$$

we denote

$$
\mathcal{F}(Z)=K(Z) P Z
$$

where

$$
K(Z)= \begin{cases}\rho^{*}+w^{*} z(t)-z^{*} w(t) & \text { if } \rho^{*}+w^{*} z(t)-z^{*} w(t) \in[0,1] \\ 0 & \text { if } \rho^{*}+w^{*} z(t)-z^{*} w(t)<0 \\ 1 & \text { if } \rho^{*}+w^{*} z(t)-z^{*} w(t)>1\end{cases}
$$

Note that, since the map $Z \mapsto w^{*} z-z^{*} w$ is Lipschitz in $X$, it is not difficult to prove that $K$ is also a Lipschitz map. Therefore, we can deduce that the map $\mathcal{F}: X \rightarrow X$ 
is Lipschitz continuous on bounded subsets of $X$ in the sense that for every $M>0$ and for every $Z_{1}, Z_{2} \in B_{M}=\{Z \in X ;\|Z\| \leqslant M\}$, we have

$$
\left\|\mathcal{F}\left(Z_{1}\right)-\mathcal{F}\left(Z_{2}\right)\right\| \leqslant C_{M}\left\|Z_{1}-Z_{2}\right\| .
$$

Using Proposition 4.3.9 from Cazenave and Haraux [6] we obtain the uniqueness and existence of a strong solution of (2.7), i.e., the first conclusion of the proposition.

Moreover, the obtained solution is smooth enough to validate the calculations done to obtain (1.16), so that

$$
\begin{aligned}
W(Z(t))+ & 2 R_{L} \int_{0}^{t}\left|I(1, \sigma)-I^{*}\right|^{2} \mathrm{~d} \sigma \\
& -2 \int_{0}^{t}\left(\rho^{*}-\rho(\sigma)\right)\left(w^{*} z(\sigma)-w(\sigma) z^{*}\right) \mathrm{d} \sigma=W\left(Z_{0}\right) \quad(t \in[0, T]) .
\end{aligned}
$$

By density, if $Z_{0} \in X$, there exists $Z_{0 n} \in \mathcal{D}(A)$ such that $Z_{0 n} \rightarrow Z_{0}$ in $X$. Denote by $Z_{n}$ the solution of

$$
\left\{\begin{array}{l}
\dot{Z}_{n}(t)=A Z_{n}(t)+\rho_{n}(t) P Z_{n}(t)+F \\
Z_{n}(0)=Z_{0 n}
\end{array}\right.
$$

where

$$
\rho_{n}(t)= \begin{cases}\rho^{*}+w^{*} z_{n}(t)-z^{*} w_{n}(t) & \text { if } \rho^{*}+w^{*} z_{n}(t)-z^{*} w_{n}(t) \in[0,1] \\ 0 & \text { if } \rho^{*}+w^{*} z_{n}(t)-z^{*} w_{n}(t)<0 \\ 1 & \text { if } \rho^{*}+w^{*} z_{n}(t)-z^{*} w_{n}(t)>1\end{cases}
$$

Applying (2.9), we have

$$
\begin{aligned}
& W\left(Z_{n}(t)\right)+2 R_{L} \int_{0}^{t}\left|I_{n}(1, \sigma)-I^{*}\right|^{2} \mathrm{~d} \sigma \\
& \quad-2 \int_{0}^{t}\left(\rho^{*}-\rho_{n}(\sigma)\right)\left(w^{*} z_{n}(\sigma)-w_{n}(\sigma) z^{*}\right) \mathrm{d} \sigma=W\left(Z_{0 n}\right) \quad\left(t \in[0, T], n \in \mathbb{N}^{*}\right) .
\end{aligned}
$$

Therefore, there exists $K_{T}>0$ such that

$$
\left\|Z_{n}(t)\right\| \leqslant K_{T} \quad\left(t \in[0, T], \quad n \in \mathbb{N}^{*}\right) .
$$

Denote

$$
Z_{m n}=Z_{n}-Z_{m}=\left[\begin{array}{c}
z_{n}-z_{m} \\
w_{n}-w_{m} \\
I_{n}-I_{m} \\
V_{n}-V_{m}
\end{array}\right] \quad(m, n \in \mathbb{N})
$$

Then

$$
\left\{\begin{array}{l}
\dot{Z}_{m n}(t)=A Z_{m n}(t)+\rho_{n}(t) P Z_{m n}(t)+\left(\rho_{n}(t)-\rho_{m}(t)\right) P Z_{m}(t) \\
Z_{m n}(0)=Z_{0 n}-Z_{0 m}
\end{array}\right.
$$

Since $A$ is dissipative and $\langle P Z, Z\rangle=0$ for every $Z \in X$, it is not difficult to prove that the solution of (2.12) satisfies

$$
\left\|Z_{m n}(t)\right\|^{2} \leqslant\left\|Z_{0 n}-Z_{0 m}\right\|^{2}+2 \int_{0}^{t}\left(\rho_{n}(s)-\rho_{m}(s)\right)\left\langle P Z_{m}(s), Z_{m n}(s)\right\rangle \mathrm{d} s .
$$


We deduce from (2.11) that

$$
\left\|Z_{m n}(t)\right\|^{2} \leqslant\left\|Z_{0 n}-Z_{0 m}\right\|^{2}+M \int_{0}^{t}\left|\rho_{n}(s)-\rho_{m}(s)\right|\left\|Z_{m n}(s)\right\| \mathrm{d} s,
$$

where $M>0$. As $\rho_{n}(t)=K\left(Z_{n}(t)\right)$ and as $K$ is a Lipschitz map, we have

$$
\left\|Z_{m n}(t)\right\|^{2} \leqslant\left\|Z_{0 n}-Z_{0 m}\right\|^{2}+M \int_{0}^{t}\left\|Z_{m n}(s)\right\|^{2} \mathrm{~d} s .
$$

Gronwall's lemma implies that

$$
\left\|Z_{m n}(t)\right\|^{2} \leqslant e^{M T}\left\|Z_{0 n}-Z_{0 m}\right\|^{2} .
$$

This implies, using (2.12), that the sequence $\left(Z_{m n}\right)$ converges to zero in $C([0, T], X)$ when $m, n \rightarrow \infty$. We have thus shown that $\left(Z_{n}\right)$ converges strongly in $C([0, T], X)$ to $Z$, which is the solution of (2.7) with $\rho$ given by (1.17) and with initial data $Z_{0}$.

Moreover, using (2.10), there exists $C>0$ such that

$$
\int_{0}^{T}\left|I_{n}(1, \sigma)\right|^{2} \mathrm{~d} \sigma \leqslant C \quad\left(n \in \mathbb{N}^{*}\right),
$$

and then $\left(I_{n}(1, \cdot)\right)_{n}$ is bounded in $L^{2}(0, T)$. Consequently, up to the extraction of a subsequence, $I_{n}(1, \cdot)$ converges weakly to $I(1, \cdot)$ in $L^{2}(0, T)$. Then, it suffices to pass to the limit in (2.10) to obtain (2.8).

We are now in position to prove Theorem 1.1.

Proof of Theorem 1.1. According to Proposition 2.2, the global existence follows from the fact that $\|Z(t)\|$ does not blow up in finite time. This fact is a direct consequence of (2.8). Moreover, we deduce (1.18) from (2.8).

\section{Stabilization result with the saturating feedback}

In this section we show that equations (1.1)-(1.7) with $\rho$ given by (1.17) define a strongly stable system.

To state properly our stability result, we introduce a semigroup $(S(t))_{t \geqslant 0}$ of nonlinear operators by setting, for each $t>0, S(t) Z_{0}=Z(t)$ where $Z$ is the solution of (2.7) and $\rho$ is given by the feedback law (1.17). To prove Theorem 1.3 we need the following lemma.

Lemma 3.1. For every $T>0$, if $Z_{0 n} \rightarrow Z_{\infty}$ in $X$, then $S(t) Z_{0 n} \rightarrow S(t) Z_{\infty}$ in $C([0, T], X)$.

Proof. Assume that $Z_{0 n} \rightarrow Z_{\infty}$ in $X$. Denote by $Z_{n}$ the solution of

$$
\left\{\begin{array}{l}
\dot{Z}_{n}(t)=A Z_{n}(t)+\rho_{n}(t) P Z_{n}(t)+F \\
Z_{n}(0)=Z_{0 n}
\end{array}\right.
$$

where

$$
\rho_{n}(t)= \begin{cases}\rho^{*}+w^{*} z_{n}(t)-z^{*} w_{n}(t) & \text { if } \rho^{*}+w^{*} z_{n}(t)-z^{*} w_{n}(t) \in[0,1] \\ 0 & \text { if } \rho^{*}+w^{*} z_{n}(t)-z^{*} w_{n}(t)<0 \\ 1 & \text { if } \rho^{*}+w^{*} z_{n}(t)-z^{*} w_{n}(t)>1 .\end{cases}
$$

By (2.8), there exists $K_{T}>0$ such that

$$
\left\|Z_{n}(t)\right\| \leqslant K_{T} \quad\left(t \in[0, T], \quad n \in \mathbb{N}^{*}\right) .
$$

Using the same ideas as in the proof of Proposition 2.2, we can show that $\left(Z_{n}\right)$ converges strongly in $C([0, T], X)$ to $Z$, which is the solution of (2.7) with $\rho$ given by (1.17) and with initial data $Z_{0}$. 
For a solution $Z \in C([0,+\infty), X)$ of (1.1)-(1.7), we introduce the Lyapunov function, which can be seen as a perturbation of the one introduced in (1.15).

$$
\begin{aligned}
\mathcal{E}_{\gamma}(t) & =\frac{1}{2}\left\|Z(t)-Z^{*}\right\|^{2}+\gamma \int_{0}^{1} x\left(I(x, t)-I^{*}\right)\left(V^{*}-V(x, t)\right) \mathrm{d} x \\
& =\frac{1}{2} W(Z(t))+\gamma \int_{0}^{1} x\left(I(x, t)-I^{*}\right)\left(V^{*}-V(x, t)\right) \mathrm{d} x,
\end{aligned}
$$

where $\gamma>0$ is a parameter that will be fixed small enough later on. The introduction of this type of Lyapunov functions goes back to Morawetz [15] in the study of the wave equation in exterior domains.

We first notice that $W(Z(\cdot))$ and $\mathcal{E}_{\gamma}$ are equivalent. More precisely, for $\gamma$ small enough $\left(\gamma<\min \left(L_{l}, C_{l}\right)\right)$, there exist two positive constants $c_{1}$ and $c_{2}$ such that

$$
c_{1} W(Z(t)) \leqslant \mathcal{E}_{\gamma}(t) \leqslant c_{2} W(Z(t)) .
$$

We have the following lemma:

Lemma 3.2. Let $\gamma>0$ be small enough. Then there exists a positive constant $\delta$, depending only on $\gamma$, such that for every $Z_{0} \in \mathcal{D}(A)$, the solution $Z$ of (1.1)-(1.7) satisfies

$$
\mathcal{E}_{\gamma}(0)-\mathcal{E}_{\gamma}(\tau) \geqslant \delta \int_{0}^{\tau}\left(\left\|V(\cdot, t)-V^{*}\right\|_{L^{2}[0,1]}^{2}+\left\|I(\cdot, t)-I^{*}\right\|_{L^{2}[0,1]}^{2}\right) \mathrm{d} t \quad(\tau>0) .
$$

Proof. Using (1.18) it follows that the solution $Z$ of (1.1)-(1.7) satisfies, for all $t \geqslant 0$,

$\frac{1}{2} \frac{\mathrm{d}}{\mathrm{d} t} \mathcal{E}_{\gamma}(t)=-R_{L}\left|I(1, t)-I^{*}\right|^{2}-\left|w^{*} z(t)-z^{*} w(t)\right|^{2}+\gamma \frac{\mathrm{d}}{\mathrm{d} t} \int_{0}^{1} x\left(I-I^{*}\right)\left(V^{*}-V\right) \mathrm{d} x$.

Integrating by parts and using (1.3)-(1.4), we obtain that

$$
\begin{aligned}
\frac{\mathrm{d}}{\mathrm{d} t} \int_{0}^{1} x\left(I-I^{*}\right)\left(V^{*}-V\right) \mathrm{d} x= & \int_{0}^{1} x \frac{\partial I}{\partial t}\left(V^{*}-V\right) \mathrm{d} x-\int_{0}^{1} x\left(I-I^{*}\right) \frac{\partial V}{\partial t} \mathrm{~d} x \\
= & -\frac{1}{L_{l}} \int_{0}^{1} x \frac{\partial V}{\partial x}\left(V^{*}-V\right) \mathrm{d} x+\frac{1}{C_{l}} \int_{0}^{1} x\left(I-I^{*}\right) \frac{\partial I}{\partial x} \mathrm{~d} x \\
= & -\frac{1}{2 L_{l}} \int_{0}^{1}\left|V-V^{*}\right|^{2} \mathrm{~d} x-\frac{1}{2 C_{l}} \int_{0}^{1}\left|I-I^{*}\right|^{2} \mathrm{~d} x \\
& +\frac{1}{2 L_{l}}\left|V(1, t)-V^{*}\right|^{2}+\frac{1}{2 C_{l}}\left|I(1, t)-I^{*}\right|^{2} .
\end{aligned}
$$

The last two formulas, combined with the facts that $V(1, t)=R_{L} I(1, t)$ and $V^{*}=$ $R_{L} I^{*}$, yield

$$
\begin{aligned}
\frac{1}{2} \frac{\mathrm{d}}{\mathrm{d} t} \mathcal{E}_{\gamma}(t)=-\frac{1}{2 L_{l}} \int_{0}^{1} & \left|V-V^{*}\right|^{2} \mathrm{~d} x-\frac{1}{2 C_{l}} \int_{0}^{1}\left|I-I^{*}\right|^{2} \mathrm{~d} x \\
& -\left(R_{L}-\frac{\gamma}{2 C_{l}}-\frac{\gamma R_{L}^{2}}{2 L_{l}}\right)\left|I(1, t)-I^{*}\right|^{2}-\left|w^{*} z(t)-z^{*} w(t)\right|^{2} .
\end{aligned}
$$

Consequently, for every $\gamma$ such that

$$
0<\gamma<\min \left\{\frac{R_{L}}{\frac{1}{2 C_{L}}+\frac{R_{L}^{2}}{2 L_{l}}}, L_{l}, C_{l}\right\},
$$

there exists $\delta>0$, depending only on $\gamma$, such that

$$
\frac{\mathrm{d}}{\mathrm{d} t} \mathcal{E}_{\gamma}(t) \leqslant-\delta\left(\left\|V(\cdot, t)-V^{*}\right\|_{L^{2}[0,1]}^{2}+\left\|I(\cdot, t)-I^{*}\right\|_{L^{2}[0,1]}^{2}\right),
$$

which implies (3.2). 
Lemma 3.3. For every $Z_{0} \in \mathcal{D}(A)$, the solution $Z$ of (1.1)-(1.7) satisfies

$$
\lim _{t \rightarrow+\infty} I(\cdot, t)=I^{*} \quad \text { and } \quad \lim _{t \rightarrow+\infty} V(\cdot, t)=V^{*} \quad \text { in } \quad L^{2}[0,1] .
$$

Proof. We first note, using (3.1) and Lemma 3.2, that the map

$$
t \mapsto L_{l}\left\|I(\cdot, t)-I^{*}\right\|_{L^{2}[0,1]}^{2}+C_{l}\left\|V(\cdot, t)-V^{*}\right\|_{L^{2}[0,1]}^{2}
$$

is in $\in L^{1}[0, \infty)$. To prove (3.3), we use a contradiction argument. We assume that $L_{l}\left\|I(\cdot, t)-I^{*}\right\|_{L^{2}[0,1]}^{2}+C_{l}\left\|V(\cdot, t)-V^{*}\right\|_{L^{2}[0,1]}^{2}$ do not tend to zero. Therefore, there exists $\epsilon>0$ and a positive increasing sequence $\left(t_{n}\right)_{n}$ with $t_{n} \rightarrow+\infty$ such that

$$
L_{l}\left\|I\left(\cdot, t_{n}\right)-I^{*}\right\|_{L^{2}[0,1]}^{2}+C_{l}\left\|V\left(\cdot, t_{n}\right)-V^{*}\right\|_{L^{2}[0,1]}^{2} \geqslant \epsilon .
$$

Using the fact that $Z \in C([0,+\infty), X)$ and the fact that the map in (3.4) is in $L^{1}[0, \infty)$ it follows that there exists a finite set $F$, such that

$$
\begin{aligned}
\left.\forall n \in \mathbb{N}^{*} \backslash F, \exists \alpha_{n} \in\right] 0, t_{n}-t_{n-1}[ \\
\qquad L_{l}\left\|I\left(\cdot, t_{n}-\alpha_{n}\right)-I^{*}\right\|_{L^{2}[0,1]}^{2}+C_{l}\left\|V\left(\cdot, t_{n}-\alpha_{n}\right)-V^{*}\right\|_{L^{2}[0,1]}^{2}=\frac{\epsilon}{2}
\end{aligned}
$$

We set

$$
\delta_{n}=\min \left\{\alpha_{n} \in\right] 0, t_{n}-t_{n-1}\left[; \alpha_{n} \text { satisfies }(3.6)\right\} \quad\left(n \in \mathbb{N}^{*}\right) .
$$

Using again the fact that the map in (3.4) lies in $L^{1}[0 ; \infty)$ and (3.7), together with the fact that $Z \in C([0,+\infty), X)$, we have

$$
\sum_{n \in \mathbb{N}^{*} \backslash F} \delta_{n}<+\infty
$$

It follows that $\delta_{n} \rightarrow 0$. Using (1.18) and (1.17), we have

$$
W\left(Z\left(t_{n}-\delta_{n}\right)\right) \geqslant W\left(Z\left(t_{n}\right)\right) \quad\left(n \in \mathbb{N}^{*}\right),
$$

and then, using (3.5) and (3.6), we obtain

$\frac{\epsilon}{2}+L\left|z\left(t_{n}-\delta_{n}\right)-z^{*}\right|^{2}+C\left|w\left(t_{n}-\delta_{n}\right)-w^{*}\right|^{2} \geqslant \epsilon+L\left|z\left(t_{n}\right)-z^{*}\right|^{2}+C\left|w\left(t_{n}\right)-w^{*}\right|^{2}$,

which implies

$$
\begin{aligned}
L\left|z\left(t_{n}-\delta_{n}\right)-z^{*}\right|^{2}+C\left|w\left(t_{n}-\delta_{n}\right)-w^{*}\right|^{2}-L\left|z\left(t_{n}\right)-z^{*}\right|^{2} & \\
& -C\left|w\left(t_{n}\right)-w^{*}\right|^{2} \geqslant \frac{\epsilon}{2}
\end{aligned}
$$

To obtain the contradiction it suffices to show that $\dot{z}$ and $\dot{w}$ are bounded in $C[0, \infty)$. Indeed, since $\delta_{n} \rightarrow 0$, this would contradict (3.8).

To show that $\dot{z}$ and $\dot{w}$ are bounded in $[0, \infty)$ we first note that, by (1.18), $z$ and $w$ are bounded in $[0, \infty)$. Therefore, using (1.1)-(1.2) and the fact that $\rho(t) \in[0,1]$, it is sufficient to show that $I(0,$.$) is bounded in [0, \infty)$. Checking this fact is the most difficult part of the proof, which is described below.

We can easily verify that $I+\sqrt{\frac{C_{l}}{L_{l}}} V$ is constant along the characteristic $t=\sqrt{C_{l} L_{l}} x$ and that $I-\sqrt{\frac{C_{l}}{L_{l}}} V$ is constant along the characteristic $t=-\sqrt{C_{l} L_{l}} x$. Consequently for every $t \geqslant \sqrt{C_{l} L_{l}}$, we have

$$
\left\{\begin{array}{l}
I(0, t)+\sqrt{\frac{C_{l}}{L_{l}}} V(0, t)=I\left(1, \sqrt{C_{l} L_{l}}+t\right)+\sqrt{\frac{C_{l}}{L_{l}}} V\left(1, \sqrt{C_{l} L_{l}}+t\right) \\
I(0, t)-\sqrt{\frac{C_{l}}{L_{l}}} V(0, t)=I\left(1,-\sqrt{C_{l} L_{l}}+t\right)-\sqrt{\frac{C_{l}}{L_{l}}} V\left(1,-\sqrt{C_{l} L_{l}}+t\right) .
\end{array}\right.
$$


Using (1.5), for every $t \geqslant \sqrt{C_{l} L_{l}}$, we have

$$
\left\{\begin{array}{l}
I(0, t)+\sqrt{\frac{C_{l}}{L_{l}}} w(t)=\left(1+R_{L} \sqrt{\frac{C_{l}}{L_{l}}}\right) I\left(1, t+\sqrt{C_{l} L_{l}}\right) \\
I(0, t)-\sqrt{\frac{C_{l}}{L_{l}}} w(t)=\left(1-R_{L} \sqrt{\frac{C_{l}}{L_{l}}}\right) I\left(1, t-\sqrt{C_{l} L_{l}}\right) .
\end{array}\right.
$$

We set

$$
f(t)=I(0, t)-\sqrt{\frac{C_{l}}{L_{l}}} V(0, t)=I(0, t)-\sqrt{\frac{C_{l}}{L_{l}}} w(t) \quad(t \geqslant 0),
$$

and

$$
\left.\alpha=\frac{1-R_{L} \sqrt{\frac{C_{l}}{L_{l}}}}{1+R_{L} \sqrt{\frac{C_{l}}{L_{l}}}} \in\right]-1,1[.
$$

It is not difficult to check (by induction over $p$ ) that for every $p \in \mathbb{N}^{*}$ and every $t \in\left[2 p \sqrt{C_{l} L_{l}},(2 p+1) \sqrt{C_{l} L_{l}}\right]$ we have

$f(t)=2 \sqrt{\frac{C_{l}}{L_{l}}} \sum_{k=1}^{p-1}\left(\alpha^{k} w\left(t-2 k \sqrt{C_{l} L_{l}}\right)\right)+\sqrt{\frac{C_{l}}{L_{l}}} \alpha^{p} w\left(t-2 p \sqrt{C_{l} L_{l}}\right)+\alpha^{p} I\left(0, t-2 p \sqrt{C_{l} L_{l}}\right)$,

whereas $f$ is given for every $t \in\left[(2 p+1) \sqrt{C_{l} L_{l}},(2 p+2) \sqrt{C_{l} L_{l}}\right]$, with $p \in \mathbb{N}$, by

$f(t)=2 \sqrt{\frac{C_{l}}{L_{l}}} \sum_{k=1}^{p}\left(\alpha^{k} w\left(t-2 k \sqrt{C_{l} L_{l}}\right)\right)+\frac{\left(1-R_{L} \sqrt{\frac{C_{l}}{L_{l}}}\right)^{p+1}}{\left(1+R_{L} \sqrt{\frac{C_{l}}{L_{l}}}\right)^{p}} I\left(1, t-(2 p+1) \sqrt{C_{l} L_{l}}\right)$.

Moreover, we know by (1.18), that $w$ is bounded on $[0, \infty)$. Consequently, since $|\alpha|<1, f$ is bounded on $[0, \infty)$ so that we have indeed shown that $I(0, \cdot)$ is bounded on $[0, \infty)$. As mentioned above, this concludes the proof of our lemma.

We are now in position to prove the main theorem of this paper.

Proof of Theorem 1.3. We assume that $Z$ is the solution of (1.1)-(1.7) with initial state $Z_{0} \in \mathcal{D}(A)$ and $\rho$ given by (1.17). By (1.18) we have

$$
W(Z(t)) \leqslant W\left(Z_{0}\right) \quad(t>0)
$$

so that there exists $C>0$ such that

$$
\|Z(t)\| \leqslant C \quad(t>0) .
$$

Therefore there exist a sequence $t_{n} \rightarrow+\infty$ and $Z_{\infty} \in X$ such that $Z\left(t_{n}\right)=S\left(t_{n}\right) Z_{0} \rightarrow$ $Z_{\infty}$ in $X$ weakly. The aim is to show that $Z_{\infty}=Z^{*}$.

We note $Z_{\infty}=\left(z_{\infty}, w_{\infty}, I_{\infty}, V_{\infty}\right)^{T}$. By Lemma $3.3, Z\left(t_{n}\right)=S\left(t_{n}\right) Z_{0} \rightarrow Z_{\infty}$ in $X$ (strongly) with

$$
I_{\infty}=I^{*}, \quad V_{\infty}=V^{*},
$$

and using (1.5), together with (1.13) and (1.14), we have

$$
w_{\infty}=w^{*} .
$$

It remains to prove that $z_{\infty}=z^{*}$.

To accomplish this goal we first denote

$$
g(Z(t))=\left|\rho^{*}-\rho(t)\right|\left|w^{*} z(t)-z^{*} w(t)\right| \quad(t \geqslant 0) .
$$


Using (1.18), we have

$$
\int_{0}^{t} g(Z(\sigma)) \mathrm{d} \sigma \leqslant W\left(Z_{0}\right) \quad(t \geqslant 0) .
$$

so that $g \in L^{1}[0, \infty)$. Let $T>0$. Using the fact that $Z\left(\tau+t_{n}\right)=S\left(\tau+t_{n}\right) Z_{0}=$ $S(\tau) S\left(t_{n}\right) Z_{0}$, we have

$$
0=\lim _{n \rightarrow \infty} \int_{t_{n}}^{T+t_{n}} g(Z(t)) \mathrm{d} t=\lim _{n \rightarrow \infty} \int_{0}^{T} g\left(S(\tau) S\left(t_{n}\right) Z_{0}\right) \mathrm{d} \tau
$$

Using Lemma 3.1, we obtain that

$$
0=\lim _{n \rightarrow \infty} \int_{t_{n}}^{T+t_{n}} g(Z(s)) \mathrm{d} s=\int_{0}^{T} g\left(S(\tau) Z_{\infty}\right) \mathrm{d} \tau \geqslant 0 .
$$

Consequently, we have

$$
\int_{0}^{T} g\left(S(\tau) Z_{\infty}\right) \mathrm{d} \tau=0
$$

We set $\widetilde{Z}(\tau)=S(\tau) Z_{\infty}=(\widetilde{z}(\tau), \widetilde{w}(\tau), \widetilde{I}(\tau, \cdot), \widetilde{V}(\tau, \cdot))^{T}$. We deduce from Lemma 3.3, (1.5) and the fact that $S\left(\tau+t_{n}\right) Z_{0}=S(\tau) S\left(t_{n}\right) Z_{0} \rightarrow S(\tau) Z_{\infty}=\widetilde{Z}(\tau)$ in $C([0, T], X)$ (see Lemma 3.1), that

$$
\widetilde{w}(\tau)=w^{*}, \quad \widetilde{I}(\tau, \cdot)=I^{*}, \quad \widetilde{V}(\tau, \cdot)=V^{*} \quad(\tau \in[0, T]) .
$$

Moreover, using (3.9), we have

$$
\left|\rho^{*}-\widetilde{\rho}(t)\right|\left|w^{*} \widetilde{z}(t)-z^{*} \widetilde{w}(t)\right|=0 \quad(t \in[0, T]) .
$$

Since, going back to (1.17), we see that $w^{*} \widetilde{z}(t)-z^{*} \widetilde{w}(t)=0$ whenever $\widetilde{\rho}(t)=\rho^{*}$, it follows that

$$
\widetilde{z}(t)=\frac{z^{*} \widetilde{w}(t)}{w^{*}}=z^{*} \quad(t \in[0, T]) .
$$

Therefore $Z\left(\tau+t_{n}\right)=S(\tau) S\left(t_{n}\right) Z_{0} \rightarrow S(\tau) Z_{\infty}=Z^{*}$ for every $\tau>0$, which concludes the proof.

Acknowledgements: The authors would like thank Professor George Weiss for fruitful discussions.

\section{References}

[1] H. Amini, M. Mirrahimi, And P. Rouchon, Stabilization of a delayed quantum system: the photon box case-study, IEEE Trans. Automat. Control, 57 (2012), pp. 1918-1930.

[2] J. M. Ball, J. E. Marsden, and M. Slemrod, Controllability for distributed bilinear systems, SIAM J. Control Optim., 20 (1982), pp. 575-597.

[3] J. M. Ball and M. Slemrod, Feedback stabilization of distributed semilinear control systems, Appl. Math. Optim., 5 (1979), pp. 169-179.

[4] K. Beauchard and M. Mirrahimi, Practical stabilization of a quantum particle in a one-dimensional infinite square potential well, SIAM J. Control Optim., 48 (2009), pp. 1179-1205.

[5] J. Buisson, H. Cormerais, And P. Richard, On the stabilization of switching electrical power converters, Proc. Hybrid Systems: Computation and Control. Zrich: Springer-Verlag, 2005. 
[6] T. Cazenave and A. Haraux, An introduction to semilinear evolution equations, vol. 13 of Oxford Lecture Series in Mathematics and its Applications, The Clarendon Press Oxford University Press, New York, 1998. Translated from the 1990 French original by Yvan Martel and revised by the authors.

[7] T. Geyer, G. Papafotiou, and M. Morari, On the optimal control of switchmode dc-dc converters, vol. 2993, Proc. Hybrid Systems: Computation and Control. Springer-Verlag, 2004, pp. 342-356.

[8] D. Liberzon, Switching in systems and control, Birkhauser, Boston, MA, 2003.

[9] J.-L. Lions, Contrôlabilité exacte, perturbations et stabilisation de systèmes distribués. Tome 1, vol. 8 of Recherches en Mathématiques Appliquées [Research in Applied Mathematics], Masson, Paris, 1988. Contrôlabilité exacte. [Exact controllability], With appendices by E. Zuazua, C. Bardos, G. Lebeau and J. Rauch.

[10] W. Littman And L. Markus, Exact boundary controllability of a hybrid system of elasticity, Arch. Rational Mech. Anal., 103 (1988), pp. 193-236.

[11] R. Loxton, K. Teo, V. Rehbock, And W. Ling, Optimal switching instants for a switched-capacitor dc/dc power converter, Automatica, 45 (2009), pp. 973 -980 .

[12] S. Mariethoz, S. Almer, M. Baja, A. Beccuti, D. Patino, A. Wernrud, J. Buisson, H. Cormerais, T. Geyer, H. Fujioka, U. Jonsson, C.-Y. Kao, M. Morari, G. Papafotiou, A. Rantzer, and P. Riedinger, Comparison of hybrid control techniques for buck and boost dc-dc converters, IEEE Transactions on Control Systems Technology, 18 (2010), pp. 1126 -1145.

[13] J. R. Mclaughlin and M. Slemrod, Scanning control of a vibrating string, Appl. Math. Optim., 14 (1986), pp. 27-47.

[14] R. D. Middlebrook And S. Cuk, A general unified approach to modeling switching-converter power stages, Proc. IEEE Power Electron. Specialists Conf. (PESC), 1976, pp. 18-34.

[15] C. S. Morawetz, Notes on time decay and scattering for some hyperbolic problems, Society for Industrial and Applied Mathematics, Philadelphia, Pa., 1975. Regional Conference Series in Applied Mathematics, No. 19.

[16] R. Naim, G. Weiss, and S. Ben-YaAkov, $H^{\infty}$ control applied to boost power converters, IEEE Transactions on Power Electronics, 12 (1997), pp. 677 - 683.

[17] S. Sanders, J. Noworolski, X. Liu, , and G. Verghese, Generalized averaging method for power conversion circuits, IEEE Trans. Power Electron., 6 (1991), pp. 251-259.

[18] Y. B. Shtessel, A. S. Zinober, And I. A. Shkolnikov, Sliding mode control of boost and buck-boost power converters using method of stable system centre, Automatica, 39 (2003), pp. 1061 - 1067.

[19] H. Sira-Ramirez, R. Perez-Moreno, R. Ortega, and M. GarciaEsteban, Passivity-based controllers for the stabilization of dc-to-dc power converters, Automatica, 33 (1997), pp. 499 - 513.

[20] M. Slemrod, Feedback stabilization of a linear control system in Hilbert space with an a priori bounded control, Math. Control Signals Systems, 2 (1989), pp. 265-285.

[21] M. Zainea, A. Van Der Schaft, And J. Buisson, Stabilizing control for power converters connected to transmission lines, Proceedings of the 2007 American Control Conference, 2007.

[22] X. Zhao And G. Weiss, Controllability and observability of a well-posed system coupled with a finite-dimensional system, IEEE Trans. Automat. Control, 56 (2011), pp. 88-99. 